\title{
Der Nävus sebaceus und seine assoziierten Tumorformen
}

\author{
Nevus Sebaceus and its Associated Tumors
}

Autor

Institut

\section{G. Wagner}

Hautklinik, Klinikum Bremerhaven Reinkenheide (Chefarzt: Dr. G. Wagner)
Bibliografie

DOI 10.1055/s-0028-1103497

Online-Publikation: 9.3.2009

Akt Dermatol 2009; 35:

379-384 ๔ Georg Thieme

Verlag KG Stuttgart · New York ISSN 0340-2541

\section{Korrespondenzadresse}

\section{Dr. Gunnar Wagner}

Klinikum Bremerhaven

Reinkenheide

Postbrookstr. 103

27574 Bremerhaven

gunnar.wagner@

klinikum-bremerhaven.de

\section{Zusammenfassung \\ $\nabla$}

In der vorliegenden Arbeit werden zunächst die heute bekannten epidemiologischen Daten, die histopathologischen Merkmale und die klinischen Entwicklungsphasen des Nävus sebaceus

\section{Einleitung \\ $\nabla$}

Der Nävus sebaceus ist histopathologisch eine komplexe Fehlbildung, die sich durch unterschiedlich zusammengesetzte adnexale Strukturen und verschiedenartige epidermale Differenzierungsstörungen auszeichnet. Unter hormonellem Einfluss kommt es im Laufe des Lebens zu qualitativen und quantitativen Veränderungen der einzelnen histopathologischen Bestandteile des Nävus. Auf der Grundlage dieser Veränderungen lassen sich unterschiedliche klinische Entwicklungsstadien definieren, wobei sich das Endstadium dieser Differenzierung durch das Auftreten assoziierter, sekundärer Tumorformen auszeichnet. Im Gegensatz zu früheren Einschätzungen handelt es sich in der überwiegenden Mehrzahl der assoziierten Tumorformen um gutartige Neoplasien, zu denen z. B. das Syringocystadenoma papilliferum oder das Trichoblastom gezählt werden. Maligne Neoplasien sind hingegen auBerordentlich selten, wobei innerhalb dieser Gruppe fast ausschließlich Basalzellkarzinome beobachtet werden. Von einer frühzeitigen, in der Kindheit oder Adoleszenz empfohlenen Exzision des Nävus sebaceus, die als Tumorprophylaxe propagiert wurde, hat die Mehrzahl der Autoren heute Abstand genommen. dargestellt. In einem zweiten Teil werden die verschiedenen assoziierten Tumorformen des Nävus sebaceus besprochen. Abschließend wird die Frage diskutiert, ob die prophylaktische Exzision des Nävus sebaceus heute noch vertretbar erscheint.

\section{Nävus sebaceus} $\nabla$

Der Nävus sebaceus wird zu den Hamartomen gezählt. Definitionsgemäß versteht man unter einem Hamartom eine tumoröse Fehlbildung, die in einem Organ umschrieben auftritt und aus ortsständigen, abnorm zusammengesetzten $\mathrm{Ge}$ webestrukturen besteht. Im Gegensatz zu einer Neoplasie, einem Tumor im eigentlichen Sinne, zeigt ein Hamartom kein autonomes Wachstum [1]. Manifestieren sich Hamartome in kutanen Strukturen, speziell im Bereich der Hautanhangsorgane, werden diese Fehlbildungen auch als organoide Nävi bezeichnet [2]. Die Häufigkeit des Nävus sebaceus in der Gesamtbevölkerung ist nicht bekannt, da epidemiologische Untersuchungen zur Prävalenz und Inzidenz bisher nicht durchgeführt worden sind. Innerhalb selektionierter dermatologischer Patientengruppen soll die Prävalenz des Nävus sebaceus $0,05-1 \%$ betragen [3]. Allerdings müssen auch diese Angaben als Schätzungen angesehen werden, da sie statistisch nicht belegt worden sind. Zur Häufigkeit des Nävus sebaceus im Biopsiematerial größerer Kliniken finden sich in der Literatur hingegen verschiedene Untersuchungen, deren Ergebnisse in der $\bigcirc$ Tab. 1 zusammengefasst sind.

Demzufolge ist der Nävus sebaceus eine hier regelmäßig vorkommende und mit einer Häufigkeit von $0,15-0,68 \%$ auch eine keineswegs als Rarität anzusehende Diagnose [4-8]. Da bei einem typischen klinischen Befund auf eine Biopsie verzichtet werden kann und besonders bei kleineren Exemplaren auch von Seiten der Pa- 
Tab. 1 Häufigkeit Nävus sebaceus im Biopsiematerial histopathologischer Sammlungen.

\begin{tabular}{|c|c|c|c|}
\hline Autoren & Präparate & Nävi sebacei & $\begin{array}{l}\text { Häufigkeit } \\
\text { (\%) }\end{array}$ \\
\hline $\begin{array}{l}\text { Brownstein, } \\
\text { Shapiro [4] }\end{array}$ & 78000 & 119 & 0,15 \\
\hline $\begin{array}{l}\text { Smolin, } \\
\text { Hundeiker [8] }\end{array}$ & 74000 & 122 & 0,16 \\
\hline $\begin{array}{l}\text { Mensing, } \\
\text { Jänner [7] }\end{array}$ & 53000 & 153 & 0,28 \\
\hline Chun et al. [5] & 64827 & 252 & 0,3 \\
\hline Jones, Heyl [6] & 22000 & 140 & 0,68 \\
\hline
\end{tabular}

tienten eine diagnostische Abklärung häufig nicht angestrebt wird, ist die Prävalenz des Nävus sebaceus in der Gesamtbevölkerung tatsächlich größer als die Häufigkeit der Diagnose im Biopsiematerial. Eine geschätzte Prävalenz von mindestens $1 \%$ erscheint dabei realistisch. Die Geschlechtsverteilung des Nävus sebaceus kann insgesamt als ausgeglichen angesehen werden, obwohl in einzelnen Studien sowohl das weibliche als auch das männliche Geschlecht bevorzugt betroffen gewesen sind $[7,8]$. Anhand der Daten von 5 größeren Untersuchungen mit jeweils mehr als 100 Nävi sebacei konnte das Geschlecht der Patienten ermittelt werden. Von den so erfassten insgesamt 836 Patienten waren 436 Frauen und 400 Männer, entsprechend einer Häufigkeitsverteilung von 1:0,92 [3,6-9]. Die typischen, bei der überwiegenden Mehrzahl der Patienten vorkommenden Prädilektionsstellen der Nävi sebacei sind der Kopf und die Halsregion. In mehreren Untersuchungen konnten über $90 \%$ der Nävi in den genannten Lokalisationen beobachtet werden, wobei der Hals mit etwa 3-7\% deutlich seltener betroffen gewesen ist als der Kopf $[6-8,10]$. In den verschiedenen Bereichen des Kopfes zeigte sich bei den gleichen Untersuchungen ein weitgehend einheitliches Verteilungsmuster. Mit 48,1-55,7\% der jeweils ausgewerteten Nävi sebacei war das Kapillitium am häufigsten betroffen, gefolgt vom Gesicht mit 22,8-39,8\% und der vereinzelt gesondert aufgeführten retroaurikulären Region mit 3,3-10,7\%. Hiervon abweichende Ergebnisse ermittelte nur die Arbeitsgruppe von Munoz-Perez [3]. Mit 210 Läsionen waren 93\% der insgesamt 226 in der Studie erfassten Nävi sebacei am Kapillitium lokalisiert. Nur 7\% der Fälle hatten sich außerhalb des behaarten Kopfes entwickelt. Als ungewöhnliche, nur in Einzelfällen vorkommende Lokalisationen des Nävus sebaceus gelten die Hand, die Ohrmuschel, der Genitalbereich und die Mundschleimhaut $[8,11-13]$.

Histopathologisch ist der Nävus sebaceus eine komplexe Fehlbildung. In unterschiedlichen Kombinationen finden sich Talgdrüsen, Haarfollikelstrukturen, ekkrine und apokrine Schweißdrüsen sowie epidermale Differenzierungsstörungen [6,7]. Im Laufe des Lebens können die einzelnen Bestandteile des Nävus sebaceus qualitative und quantitative Veränderungen erfahren, die dann auch das klinische Bild des Nävus beeinflussen [11]. Mehregan und Pinkus beschrieben drei mögliche Entwicklungsstadien des Nävus sebaceus [9]. Typischerweise zeigt sich der Nävus sebaceus bereits bei der Geburt als plaqueförmiger, flach erhabener, hautfarbener, rötlich oder gelblich tingierter Tumor. Neben runden oder ovalen, meist münzgroßen Läsionen finden sich lineare oder systematisierte Manifestationen $[6,8]$. Beim Auftreten am behaarten Kopf ist zunächst häufig nur eine umschriebene Alopezie erkennbar. Das histopathologi-

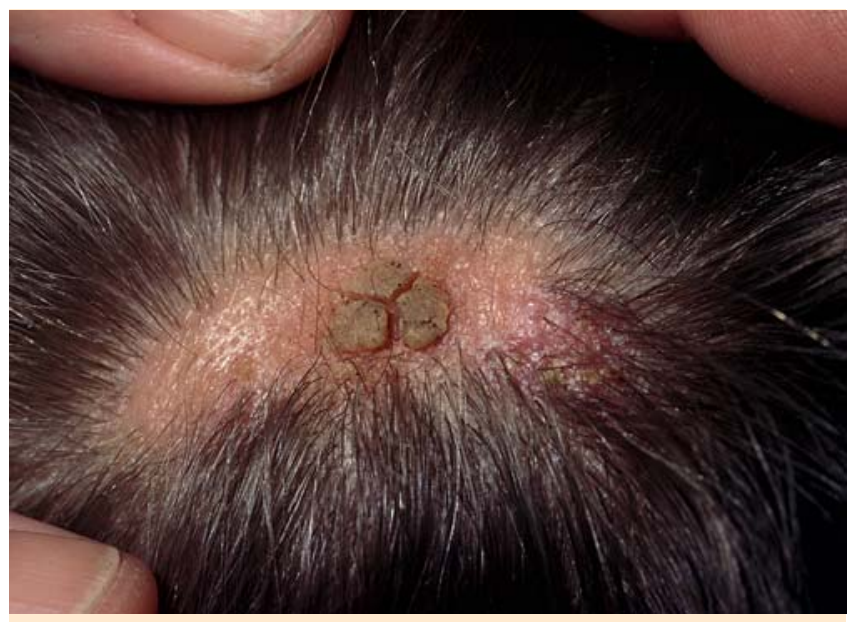

Abb. 1 Verrucae vulgares.

sche Korrelat des Nävus sebaceus besteht in der Kindheit aus einer geringfügig verbreiterten Epidermis, aus unreifen Talgdrüsen und Infundibulumstrukturen, während rudimentäre Haarwurzeln nur vereinzelt beobachtet werden können. Mit Beginn der Pubertät kommt es unter dem Einfluss hormoneller Veränderungen zu einer ausgeprägten Akanthose und Papillomatose der Epidermis, zu einer Größenzunahme und Vermehrung der Talgdrüsen sowie zu einem vermehrten Auftreten apokriner und ekkriner Drüsenstrukturen. Diese histopathologischen Veränderungen führen klinisch zu einer Volumenzunahme und zu einer kleinpapulösen, bisweilen gekörnt wirkenden oder bei ausgeprägter Epithelhyperplasie auch zu einer verrukösen Oberflächenstruktur. Im Bereich großer Follikelostien zeigen sich komedonenartige Hornpfröpfe. Der Farbton der Nävi sebacei kann nun auch graue oder dunkelbraune Färbungen annehmen. Der Palpationsbefund ist in der Regel derb oder hart, nur selten hingegen von weicher Konsistenz. Das dritte Stadium der Nävusentwicklung zeichnet sich durch das Auftreten assoziierter Tumorformen aus $[3,6,7,11,14-16]$.

\section{Assoziierte Tumorformen}

Die Häufigkeit einer sekundären Tumorentstehung innerhalb eines Nävus sebaceus kann aufgrund verschiedener Faktoren nicht zuverlässig angegeben werden. Zunächst fehlen statistisch gesicherte Angaben zur Prävalenz des Nävus sebaceus, sodass man auf Schätzungen angewiesen ist. Darüber hinaus dürften insbesondere erwachsene Patienten mit einem Nävus sebaceus in den meisten Fällen erst dann einen Arzt aufsuchen, wenn es bereits zu einer sekundären Tumorentwicklung gekommen ist. Da die assoziierten Tumorformen in der Regel exzidiert werden und die Einschätzung ihrer Häufigkeit auf der Auswertung histopathologischer Sammlungen beruht, entsteht der Eindruck, dass assoziierte Tumorformen häufiger vorkommen als dies tatsächlich der Fall ist. Auch die Frage, welche Diagnosen als assoziierte Tumorformen des Nävus sebaceus angesehen werden können, beeinflusst die statistische Auswertung der Häufigkeit. So werden von einigen Autoren virale Warzen ( Abb.1) aufgeführt, obwohl man diese histogenetisch nicht zu den assoziierten Tumorformen zählen kann $[10,17]$. Andere Autoren verwenden die Diagnose „Sebomatricoma“, einen Terminus, der als Entität nicht zu definieren ist und allenfalls als Sammelbegriff für benigne 
Tab. 2 Häufigkeit assoziierter Tumorformen des Nävus sebaceus.

\begin{tabular}{|c|c|c|c|}
\hline Autoren & $\begin{array}{l}\text { Nävi } \\
\text { sebacei }\end{array}$ & $\begin{array}{l}\text { Assoziierte } \\
\text { Tumorformen }\end{array}$ & $\begin{array}{l}\text { Häufigkeit } \\
\text { (\%) }\end{array}$ \\
\hline Chun et al. [5] & 165 & 9 & 5,4 \\
\hline Cribier et al. [10] & 596 & 100 & 16,9 \\
\hline $\begin{array}{l}\text { Munoz-Perez } \\
\text { et al. [3] }\end{array}$ & 226 & 49 & 21,6 \\
\hline $\begin{array}{l}\text { Mensing, } \\
\text { Jänner [7] }\end{array}$ & 153 & 37 & 25,0 \\
\hline Linse et al. [19] & 200 & 57 & 28,5 \\
\hline Jones, Heyl [6] & 140 & 50 & 35,7 \\
\hline Jaqueti et al. [11] & 155 & 73 & 47,0 \\
\hline
\end{tabular}

Tumorformen mit sebozytärer Differenzierung benutzt werden kann $[11,18]$. Auch die Hyperplasien verschiedener Gewebestrukturen sind von einzelnen Autoren in den Statistiken berücksichtigt worden, während andere Arbeitsgruppen ausschließlich definierte Tumorformen in ihre Auswertung aufgenommen haben. Es verwundert daher nicht, dass die Angaben zur Häufigkeit assoziierter Tumoren des Nävus sebaceus deutliche Unterschiede aufweisen mit einer Variationsbreite von 5,4-47,0\% (๑ Tab. 2).

Aus den besagten Gründen ergeben sich auch bei den Angaben zur Häufigkeit der einzelnen assoziierten Tumorformen des Nävus sebaceus teilweise deutliche Unterschiede. Darüber hinaus wurden bei früheren Untersuchungen die Trichoblastome nicht von den Basalzellkarzinomen abgegrenzt, sodass deren Häufigkeit im Vergleich mit späteren Untersuchungen überschätzt wurde $[11,19]$. Der von einzelnen Autoren als eigenständige Diagnose verwendete Begriff der basaloiden Epidermisproliferation hat die Häufigkeitsangaben der einzelnen Tumorformen ebenfalls beeinflusst, zumal diese Proliferationen teilweise als benigne, teilweise aber auch als initiale Basalzellkarzinome eingeordnet worden sind $[6,19]$. Auch dieser Umstand hat dazu beigetragen, dass die Prävalenzraten der Nävus sebaceus assoziierten Basalzellkarzinome zu hoch angegeben worden sind. Die sekundären Tumorformen des Nävus sebaceus lassen sich in benigne und maligne Neoplasien einteilen. Unter Berücksichtigung der Probleme der histogenetischen Zuordnung und der histopathologischen Einteilung der assoziierten Tumoren kann heute davon ausgegangen werden, dass mit 80-95\% der überwiegende Anteil der Neubildungen gutartigen Tumorformen zugeordnet werden kann $[3,10]$. Auch das vollständige Fehlen maligner Tumoren ist bei einzelnen Untersuchungen der letzten Jahre beschrieben worden $[5,20]$. Hingegen sind Häufigkeitsangaben maligner Neoplasien von bis zu $40 \%$ vermutlich auf die nur sehr geringen Fallzahlen dieser Untersuchungen zurückzuführen [17]. Innerhalb der Gruppe der gutartigen Neoplasien des Nävus sebaceus wurden von verschiedenen Arbeitsgruppen das Syringocystadenoma papilliferum und das Trichoblastom am häufigsten beobachtet ( Abb. 2-4). Von insgesamt 41 benignen Tumoren konnten Munoz-Perez et al. in 29 Fällen ein Syringocystadenoma papilliferum und bei weiteren 10 Patienten ein Trichoblastom diagnostizieren [3]. Cribier et al. fanden bei 30 von 81 benignen Tumorformen ein Syringocystadenoma papilliferum und mit 28 Fällen fast gleich viele Trichoblastome [10]. Von 33 gutartigen Neoplasien, die von Jaqueti et al. ausgewertet werden konnten, erwiesen sich 12 als ein Trichoblastom und 10 als ein Syringocystadenoma papilliferum [11]. In einer kleineren Gruppe von 9 assoziierten Tumoren des Nävus sebaceus konnten

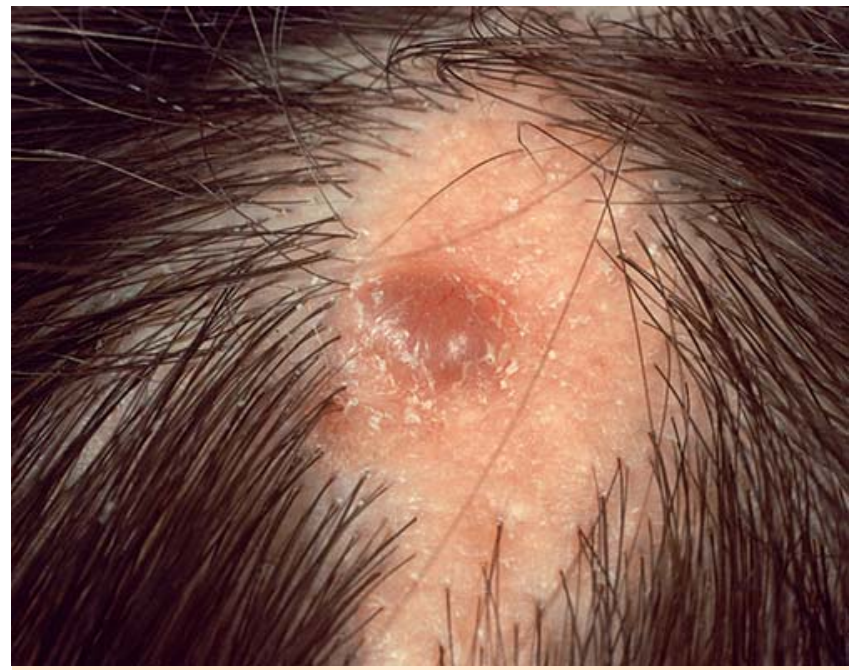

Abb. 2 Trichoblastom.

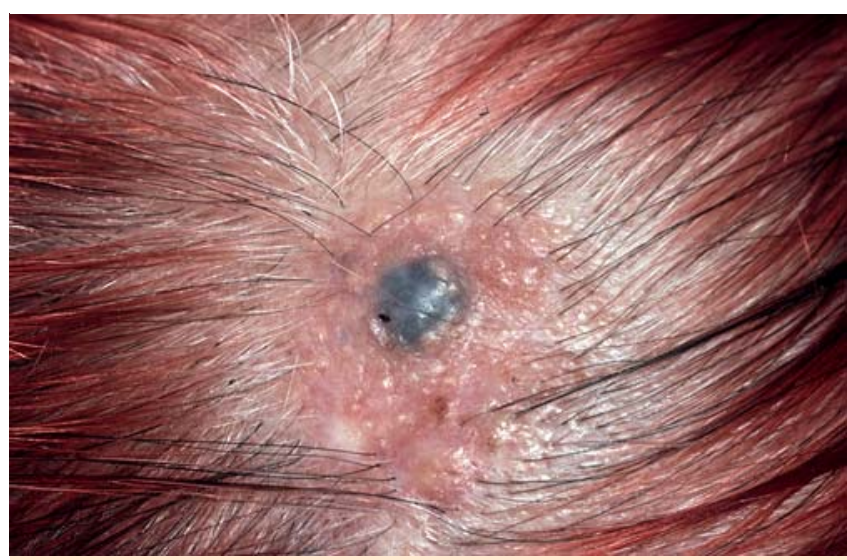

Abb. 3 Pigmentiertes Trichoblastom.

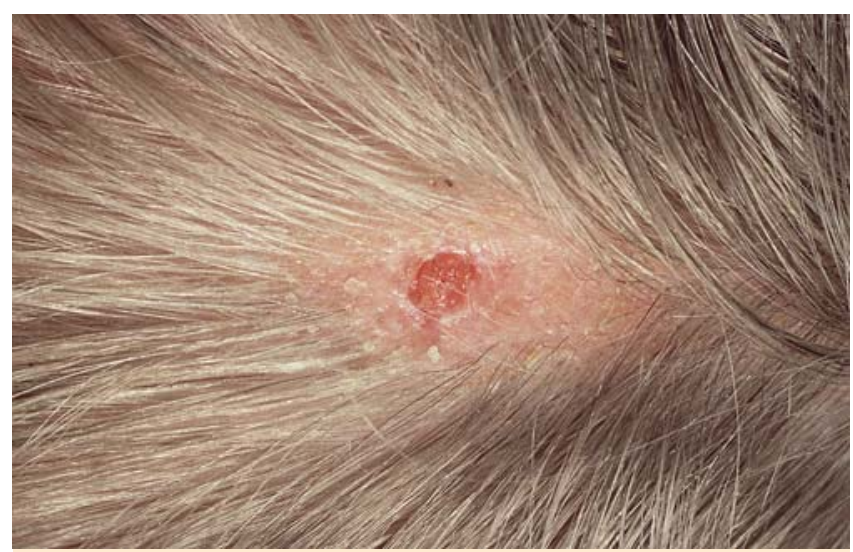

Abb. 4 Syringocystadenoma papilliferum.

Chun et al. bei 5 Fällen ein Trichoblastom und bei 3 ein Syringocystadenoma papilliferum nachweisen [5]. Auch bei den Untersuchungen von Mensing und Jänner sowie von Jones und Heyl war das Syringocystadenoma papilliferum die häufigste benigne Neoplasie des Nävus sebaceus. Mensing und Jänner fanden den Tumor in 19 von 22 Fällen. Bei Jones und Heyl war das Syringocystadenoma papilliferum mit 27 Fällen sogar die alleinige Diagnose der gutartigen Tumorformen [6]. Beide Autorengruppen di- 
Tab. 3 Benigne assoziierte Tumorformen des Nävus sebaceus.

$\begin{array}{ll}\text { Gehäuftes Vorkommen } & \\ \text { Syringocystadenoma papilliferum } & {[10,11]} \\ \text { Trichoblastom } & {[10,11]} \\ \text { Tricholemmom } & {[10,17]} \\ \text { Einzelfälle } & \\ \text { Hidrozystom (Zystadenom) } & {[3,5,11]} \\ \text { Hidradenom } & {[19]} \\ \text { Porom } & {[11]} \\ \text { Spiradenom } & {[34]} \\ \text { Syringom } & {[6,35] 56} \\ \text { Desmoplastisches Tricholemmom } & {[11,37]} \\ \text { Pilomatrixom } & {[7]} \\ \text { Osteom } & {[6,38]} \\ \text { Neurinom } & {[7]} \\ \text { Piloleiomyom } & {[39,40]} \\ \text { NZN } & {[10]} \\ \text { Xanthogranulom } & {[41]} \\ \text { Seborrhoische Keratose } & {[10,17]} \\ \text { Verruca vulgaris } & {[17]}\end{array}$

agnostizierten noch kein Trichoblastom, weil diese Tumorform zum damaligen Zeitpunkt noch nicht als Entität definiert war. Neben den Syringocystadenomata papillifera und den Trichoblastomen sind weitere benigne Neoplasien als assoziierte Tumorformen des Nävus sebaceus beobachtet worden. Von einzelnen Ausnahmen abgesehen, wie z.B. dem Tricholemmom, sind diese weiteren Tumorformen nur gelegentlich in größeren histopathologischen Studien oder in vereinzelten Kasuistiken beschrieben worden. Eine Übersicht findet sich in der $\bullet$ Tab. 3.

Die Entwicklung einer malignen Neoplasie innerhalb eines Nävus sebaceus wird heute im Gegensatz zu früheren Einschätzungen als ein seltenes Ereignis angesehen. Bei einer Analyse von insgesamt 6 Untersuchungen, die zwischen 1962 und 1985 publiziert worden waren, konnten Cribier et al. für das Nävus sebaceus assoziierte Basalzellkarzinom Häufigkeitsangaben zwischen 6,5 und 50\% ermitteln [10]. Linse et al. kamen aufgrund eigener Untersuchungen und einer Literaturauswertung zu dem Ergebnis, dass das Risiko, innerhalb eines Nävus sebaceus ein Basalzellkarzinom zu entwickeln, mit $17,8 \%$ beachtlich groß sei [19]. Mensing und Jänner sowie Smolin und Hundeiker diagnostizierten Basalzellkarzinome bei 12,5 bzw. 11,5\% der von ihnen beobachteten Nävi sebacei $[7,8]$. Alle genannten Untersuchungen haben jedoch noch nicht das Trichoblastom als eigenständigen und insbesondere von den Basalzellkarzinomen abzugrenzenden Tumor berücksichtigt. Dabei ist die klinische Morphologie der beiden Tumorformen weitgehend identisch, sodass eine zuverlässige klinische Diagnose nur in Ausnahmefällen möglich sein wird. Sowohl die Trichoblastome als auch die Basalzellkarzinome zeichnen sich innerhalb eines Nävus sebaceus durch derbe, vereinzelt pigmentierte Papeln und Knoten aus, wobei Ulzerationen möglicherweise als klinisches Merkmal der Basalzellkarzinome gewertet werden können $[3,10]$. Eine sichere diagnostische Abgrenzung der beiden Tumorformen ist jedoch nur anhand histopathologischer Befunde möglich, wobei auch hier große morphologische Ähnlichkeiten bestehen. Der heute verwendete und in dieser Form von Ackerman et al. geprägte Begriff des Trichoblastoms beruht auf den histopathologischen Befunden einer symmetrischen Tumorarchitektur mit vertikalem Wachstum, basaloiden Zellverbänden mit verschiedenen Wachstumsmustern, umschriebenen Zonen mit follikulärer Dif-

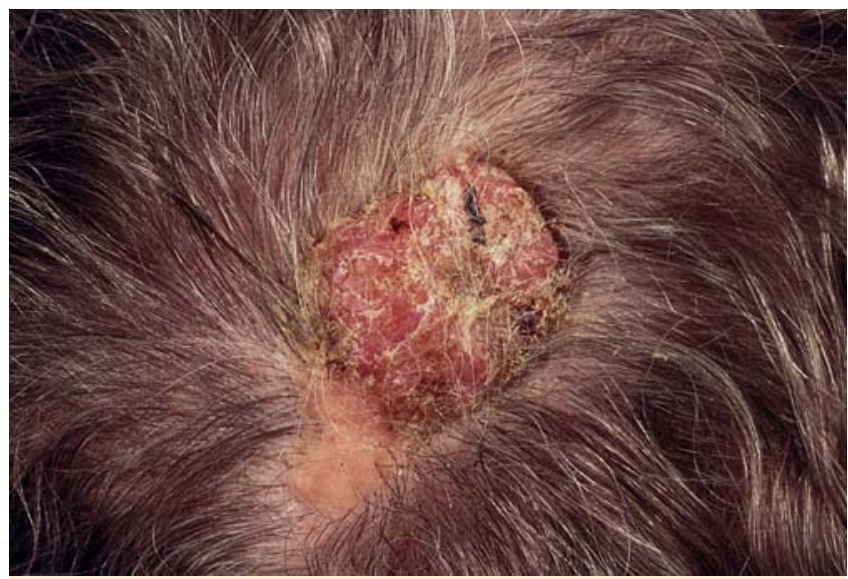

Abb. 5 Basalzellkarzinom.

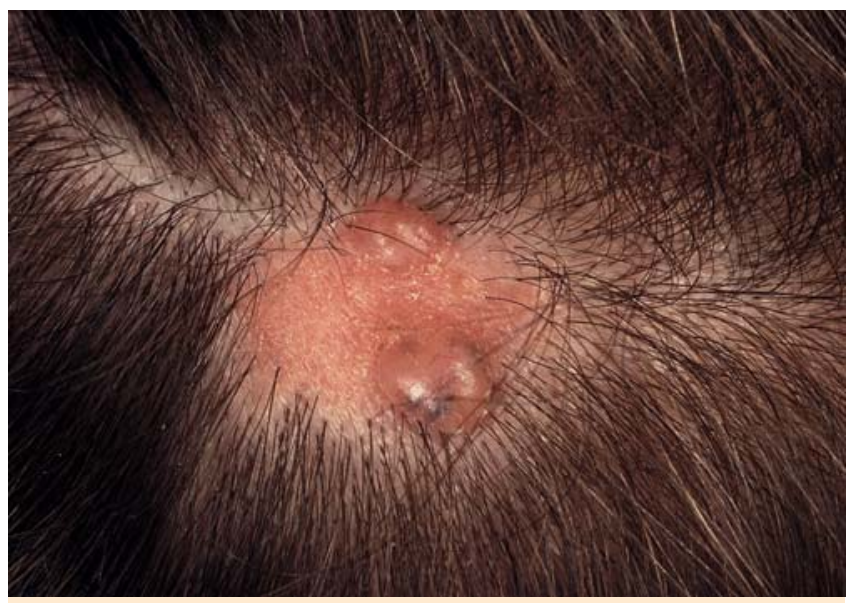

Abb. 6 Multizentrisches Basalzellkarzinom.

ferenzierung und einer artefiziellen Spaltbildung zwischen dem Stroma des Tumors und dem Bindegewebe der Dermis [21].

Unter Berücksichtigung dieser Kriterien wurden zahlreiche in früheren Untersuchungen als Basalzellkarzinome diagnostizierte Tumoren später als Trichoblastome eingeordnet [10,11]. In neueren Untersuchungen der letzten Jahre, bei denen die Autoren das Trichoblastom vom Basalzellkarzinom abgegrenzt haben, diagnostizierten die Arbeitsgruppen von Chun und von Jaqueti keine Basalzellkarzinome, während Cribier et al. die Häufigkeit des Tumors mit 0,8\% und Munoz-Perez et al. eine solche von 3,5\% angaben, bezogen auf alle jeweils untersuchten Nävi sebacei $[3,5,10,11]$. Innerhalb der Gruppe der assoziierten malignen Neoplasien des Nävus sebaceus ist das Basalzellkarzinom somit der häufigste Tumor, insgesamt ist eine Entstehung jedoch ein seltenes Ereignis ( $\bullet$ Abb. 5 und 6). Weitere Malignome, die nur ganz vereinzelt als Raritäten in einem Nävus sebaceus beobachtet wurden, sind Plattenepithelkarzinome einschließlich vereinzelter Keratoakanthome ( Abb. 7), verschiedene Adnexkarzinome, z.B. das ekkrine Porokarzinom, apokrine Karzinome und Talgdrüsenkarzinome, sowie maligne Melanome und ein Leiomyosarkom [13,22 - 27]. Die Prognose der Nävus sebaceus assoziierten Karzinome ist zweifelhaft. Bei Plattenepithelkarzinomen, ekkrinen Porokarzinomen und apokrinen Karzinomen wurden wiederholt lymphogene oder generalisierte Metastasierungen beschrieben $[23,27,28]$. Neben der Entwicklung einer 


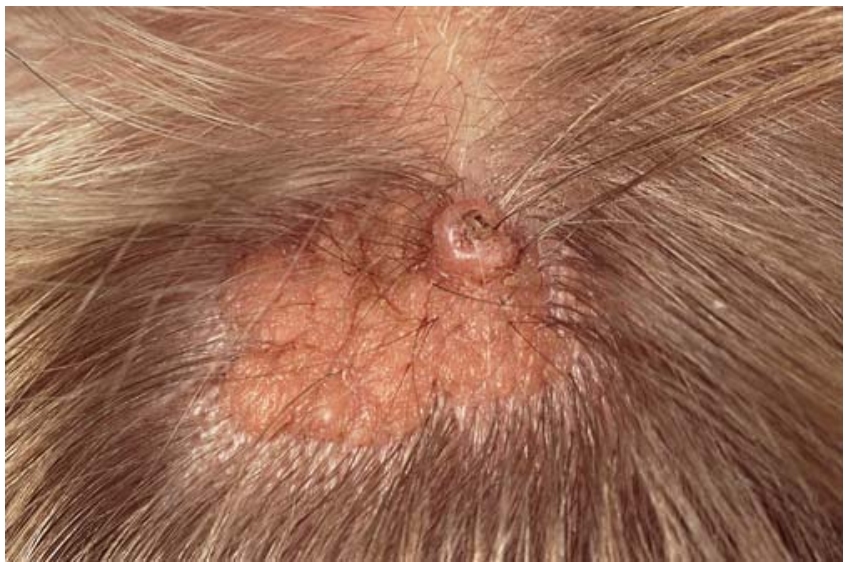

Abb. 7 Keratoakanthom.

singulären Neoplasie ist auch das gleichzeitige Auftreten verschiedener Tumorformen innerhalb eines Nävus sebaceus möglich. In der Mehrzahl dieser Fälle fanden sich zwei oder mehr gutartige Tumoren, wobei jedoch auch Kombinationen mit malignen Neoplasien beobachtet worden sind [13,17,29,30]. Von den 37 Nävi sebacei, die in der Untersuchung von Mensing und Jänner assoziierte Tumorformen aufwiesen, konnten bei 6 Fällen jeweils zwei verschiedene Neoplasien nachgewiesen werden [7]. Welches Ausmaß die Tumorentstehung im Einzelfall annehmen kann, zeigt eine Kasuistik von Miller et al. Die Autoren konnten in einem Nävus sebaceus nebeneinander ein Talgdrüsenkarzinom, ein Basalzellkarzinom, ein Trichoadenom, ein Trichoblastom und ein Syringocystadenoma papilliferum histopathologisch nachweisen [31].

\section{Nåvus sebaceus - Prophylaktische Exzision?}

Die Frage, ob man einen Nävus sebaceus bereits in der Kindheit oder der Adoleszenz prophylaktisch exzidieren sollte, ist in der Literatur kontrovers diskutiert worden. Zahlreiche Studien, die in den 1960er- bis 1980er-Jahren publiziert wurden, beschrieben mit Häufigkeiten zwischen 6,5 und 50\% einen hohen Anteil Basalzellkarzinome innerhalb der untersuchten Nävi sebacei [10]. Vor dem Hintergrund dieser Häufigkeitsangaben wurden von Linse et al. der Vorschlag zur Diskussion gestellt, den Nävus sebaceus als fakultative Präkanzerose einzuordnen und ihn im 2. Lebensjahrzehnt vor Beginn seiner Proliferationstendenz prophylaktisch zu exzidieren [19]. Auch andere Arbeitsgruppen sprachen sich für die prophylaktische Exzision des Nävus sebaceus aus, ohne allerdings von einer fakultativen Präkanzerose zu sprechen $[8,32,33]$. Nachdem sich das Trichoblastom als eigenständige Tumorform etabliert hatte und darüber hinaus Basalzellkarzinome auch rückblickend als Trichoblastome eingeordnet werden konnten, hat sich die Einstellung zur prophylaktischen Exzision des Nävus sebaceus grundlegend verändert. Bei einer Häufigkeit der Nävus sebaceus assoziierten Basalzellkarzinome von $0,8-3,5 \%$ und einem mittleren Alter der betroffenen Patienten von etwa 50 Jahren erscheint die prophylaktische Exzision des Nävus nicht gerechtfertigt $[3,10]$. Besonders die bisweilen propagierte prophylaktische Exzision in der Kindheit oder bei Jugendlichen ist nicht mehr vertretbar, nachdem in einer retrospektiven Untersuchung von Santibanez-Gallerani et al. bei 658 Kindern und Jugendlichen im Alter zwischen 2 und
16 Jahren kein einziges Basalzellkarzinom in einem Nävus sebaceus nachgewiesen werden konnte [20]. Allerdings ist auch in der neueren Literatur der grundsätzliche Verzicht zur prophylaktischen Exzision des Nävus sebaceus nicht gänzlich unumstritten. Mit dem Hinweis auf die Entstehungsmöglichkeit adnexaler Karzinome wird von einzelnen Autoren die frühzeitige Exzision des Nävus sebaceus weiterhin empfohlen [31,34]. Unterstellt man eine geschätzte Prävalenz des Nävus sebaceus in der Gesamtbevölkerung von mindestens $1 \%$ und berücksichtigt man gleichzeitig, dass adnexale Karzinome nur sehr selten als Raritäten in einem Nävus sebaceus beschrieben worden sind, kann hier sicher nicht von einem erhöhten Tumorrisiko ausgegangen werden. Der Autor selbst hat in 30 Berufsjahren etwa 10-15 Adnexkarzinome diagnostiziert. Keines dieser Karzinome war in einem Nävus sebaceus aufgetreten.

In unserer Klinik wird der Nävus sebaceus nicht mehr prophylaktisch exzidiert. Die Eltern der betroffenen Kinder werden in verständlicher und vernünftiger Form über die Natur des Nävus sebaceus und seine seltenen Komplikationen informiert, ohne dass unnötige Ängste provoziert werden. Auch eine routinemäßige Überwachung der Kinder erfolgt nicht.

\section{Abstract}

\section{Nevus Sebaceus and its Associated Tumors \\ $\nabla$}

The following publication first describes the today well-known epidemiological data, histopathological characteristics as well as the clinical developmental stages of nevus sebaceus. The second part contributes to the discussion about the different types of associated tumors of nevus sebaceus. Finally the presentation focuses the question of a prophylactic excision of nevus sebaceus in modern medicine.

\section{Literatur}

1 Eder M, Gedigk P. Lehrbuch der Allgemeinen Pathologie und der Pathologischen Anatomie. 29. Aufl. Berlin: Spinger, 1975

2 Wodniansky P. Die gutartigen Neubildungen des Integuments. In: Jadassohn J (Hrsg). Handbuch der Haut- und Geschlechtskrankheiten. Ergänzungswerk III/A. Berlin: Springer, 1975

3 Munoz-Perez MA, Garcia-Hernandez JJ, Rios F et al. Sebaceus naevi: a clinicopathologic study. J Eur Acad Dermatol Venereol 2002; 16: $319-324$

4 Brownstein MH, Shapiro L. The pilosebaceous tumors. Int J Dermatol 1977; 147: 177- 183

5 Chun K, Vazquez M, Sanchez JL. Nevus sebaceus: clinical outcome and considerations for prophylactic excision. Int J Dermatol 1995; 34: $538-541$

6 Jones EW, Heyl T. Naevus sebaceus. A report of 140 cases with special regard to the development of secondary malignant tumours. Br J Dermatol 1970; 82: 99-117

7 Mensing H, Jänner M. Naevus sebaceus Jadassohn und Syringocystadenoma papilliferum. Hautarzt 1981; 32: 130-135

8 Smolin Th, Hundeiker M. Plattenepithel- und Basalzellkarzinome im Naevus sebaceus (Jadassohn). Z Hautkr 1986; 61: 267-282

9 Mehregan HA, Pinkus H. Life history of organoid naevi. Arch Dermatol 1965; 91: $574-588$

10 Cribier B, Scrivener $Y$, Grosshans E. Tumors arising in nevus sebaceus: a study of 596 cases. J Am Acad Dermatol 2000; 42: 263 - 268

11 Jaqueti G, Requena L, Yus ES. Trichoblastom is the most common neoplasm developed in nevus sebaceus of Jadassohn. Am J Dermatopathol 2000; 22: $108-118$

12 Morency R, Labelle H. Nevus sebaceus of Jadassohn: a rare oral presentation. Oral Surg Oral Med Oral Path 1987; 64: 460-462 
13 Misagio N, Kodera H, Narisawa Y. Sebaceous carcinoma, trichoblasto$\mathrm{ma}$, and sebaceoma with features of trichoblastoma in nevus sebaceus. Am J Dermatopathol 2001; 23: 456-462

$14 \mathrm{Kerl} \mathrm{H,} \mathrm{Garbe} \mathrm{C,} \mathrm{Cerroni} \mathrm{L,} \mathrm{Wolff} \mathrm{HH.} \mathrm{Histopathologie} \mathrm{der} \mathrm{Haut.} \mathrm{1.} \mathrm{Auf.}$ Berlin: Springer, 2003

15 Rupec M, Horn W. Benigne Tumoren der Haut. In: Korting GW (Hrsg). Dermatologie in Praxis und Klinik. Stuttgart: Thieme, 1981

16 Steigleder GK, Cortes AC. Verhalten der Talgdrüsen im Talgdrüsennaevus während des Kindesalters. Arch Klin Exp Dermatol 1970; 239 : $323-328$

17 Baykal C, Buyukbabani N, Yazganoglu KD et al. Mit Naevus sebaceus assoziierte Tumoren. JDDG 2006; 4: 28 - 31

18 Yus ES, Requena L, Simon P et al. Sebomatricoma: a unifying term that encompasses all benign neoplasms with sebaceous differentiation. Am J Dermatopathol 1995; 17: 213 - 221

19 Linse R, Hadlich J, Ehlers $E$ et al. Sekundärproliferationen auf Naevus sebaceus. Dermatol Mon schr 1987; 173: 153 - 157

20 Santibanez-Gallerani A, Marshall D, Duarte AM et al. Should nevus sebaceus of Jadassohn in children be excised? A study of 757 cases, and literature review. J Craniofac Surg 2003; 14: 658-660

21 Ackerman AB, de Viragh PA, Chongchitnant N. Neoplasms with follicular differentiation. Philadelphia: Lea u. Febiger, 1993

22 Abe S, Yamamoto Y, Uno S et al. Malignant melanoma arising in a sebaceous nevus of scalp. Br J Plast Surg 2003; 56: 171 - 173

23 Domingo J, Helwig EB. Malignant neoplasms associated with nevus sebaceus of Jadassohn. J Am Acad Dermatol 1979; 1: 545-556

24 Duncan $A$, Wilson $N$, Leonard $N$. Squamous cell carcinoma developing in a nevus sebaceous of Jadassohn. Am J Dermatopathol 2008; 30: $269-270$

25 Oehlschlaegel G, Rakoski J. Keratoakanthom auf Naevus sebaceus. Z Hautkr 1981; 56: 1585 - 1595

26 Premalata CS, Kumar RV, Malathi M et al. Cutaneous leiomyosarcoma, trichoblastoma, and syringocystadenoma papilliferum arising from nevus sebaceus. Int J Dermatol 2007; 46: 306-308

27 Tarkahn II, Domingo J. Metastasizing eccrine porocarcinoma developing in a sebaceous nevus of Jadassohn. Report of a case. Arch Dermatol $1985 ; 121: 413-415$
28 Schirren CG, Pfirstinger H. Zur Entwicklung von Plattenepithel-Carcinomen auf dem Boden des Naevus sebaceus. Hautarzt 1963; 14: $397-401$

29 De Giorgi V, Massi D, Trez E et al. Multiple pigmented trichoblastomas and syringocystadenoma papilliferum in naevus sebaceous mimicking a malignant melanoma: a clinical dermoscopic-pathological case study. Br J Dermatol 2003; 149: 1067-1070

30 Wiesner M, Woenckhaus C, Hein Ket al. Syringocystadenoma papilliferum und Basalzellkarzinom der Kopfhaut. Akt Dermatol 2001; 27: $70-73$

31 Miller CJ, Ioffreda MD, Billingsley EM. Sebaceous carcinoma, basal cell carcinoma, trichoadenoma, trichoblastoma, and syringocystadenoma papilliferum arising within a nevus sebaceus. Dermatol Surg 2004; 30: $1546-1549$

32 Constant E, Davis DG. The premalignant nature of the sebaceous nevus of Jadassohn. Plast Reconstr Surg 1972; 50: 257-259

33 Lillis PJ, Ceilley RI. Multiple tumors arising in nevus sebaceous. Cutis 1979; 23: 310-314

34 Shapiro M, Johnson B, Witmer $W$ et al. Spiradenoma arising in a nevus sebaceus of Jadassohn. Am J Dermatopathol 1999; 21: $462-467$

35 Morioka S. The natural history of nevus sebaceus. J Cutan Pathol 1985; 12: $200-213$

36 Grund JL, Folan DW. Syringocystadenoma papilliferum, nevus sebaceus and basal-cell epithelioma (with features of epithelioma adenoides cysticum and syringoma): their concurrent presence in a single tumor. Urol Cutan Rev 1951; 55: 707

37 Roson E, Gomez Centeno P, Sanchez-Aguilar D et al. Desmoplastic trichilemmoma arising within a nevus sebaceus. Am J Dermatopathol 1998; 20: 495 - 497

38 Coskey RJ, Mehregan AH. Metaplastic bone formation in an organoid nevus. Arch Dermatol 1970; 102: 233

39 Burden PA, Gentry RH, Fitzpatrick JE. Piloleiomyoma arising in an organoid nevus: a case report and review of the literature. J Dermatol Surg Oncol 1987; 13: $1213-1218$

40 Serpas de Lopez RM, Hernandez-Perez E. Jadassohn's sebaceous nevus. J Dermatol Surg Oncol 1985; 11: 68-72

41 Chang SE, Choe JH, Sung KJ et al. A case of juvenile xanthogranuloma arising on a nevus sebaceus. Am J Dermatopathol 2001; 23: 347-350 